\title{
Tracking Cell Signals in Fluorescent Images
}

Victoria Gor

Jet Propulsion Laboratory,

Instruments and Science Data Systems

victoria.gor@jpl.nasa.gov

Michael Elowitz

Department of Biology and Applied Physics, Caltech

melowitz@caltech.edu

\author{
Tigran Bacarian \\ Institute for Genomics and Bioinformatics, \\ and Department of Computer Science, \\ University of California, Irvine \\ tbacaria@uci.edu \\ Eric Mjolsness \\ Institute for Genomics and Bioinformatics, \\ and Department of Computer Science, \\ University of California, Irvine \\ emj@uci.edu.
}

\begin{abstract}
In this paper we present the techniques for tracking cell signal in GFP (Green Fluorescent Protein) images of growing cell colonies. We use such tracking for both data extraction and dynamic modeling of intracellular processes. The techniques are based on optimization of energy functions, which simultaneously determines cell correspondences, while estimating the mapping functions. In addition to spatial mappings such as affine and Thin-Plate Spline mapping, the cell growth and cell division histories must be estimated as well. Different levels of joint optimization are discussed.

The most unusual tracking feature addressed in this paper is the possibility of one-to-two correspondences caused by cell division. A novel extended softassign algorithm for solutions of one-to-many correspondences is detailed in this paper. The techniques are demonstrated on three sets of data: growing bacillus Subtillus and e-coli colonies and a developing plant shoot apical meristem. The techniques are currently used by biologists for data extraction and hypothesis formation.
\end{abstract}

\section{Introduction}

\subsection{Motivation}

The deployment of in vivo live confocal microscopy has enabled scientists to capture gene expression data and begin to create computational models of developing cellular organisms. Such systems include intracellular molecular regulation networks combined with intercellular signals and transport, cell growth and proliferation, and mechanical interactions between cells, resulting in complex interaction networks with the ability to control the development of multicellular organisms.

The amount and quality of collected expression data is significant enough that scientists are able to hypothesize many of the underlying control circuits, but the experimental data of important molecular players and interactions are most often incomplete, and additional hypotheses are needed to explain their spatial and dynamical behavior [1]. Mathematical modeling provides a powerful method for describing and testing hypotheses about developmental biological systems. Not only can hypotheses be tested to see if they account for the observed data but predictions can be made for new experiments.

The data provided by the confocal imaging technique is available in the form of an image time series, quantification of which is essential to creation of viable models. Different image processing algorithms are used to extract cell compartments and GFP fluorescence intensities within individual cells $[2,3]$. It is usually assumed that the GFP intensity is linearly related to the amount of protein, and hence the average intensity within a cell is interpreted as a relative protein concentration.

Once the cell boundaries and the signal within cells is extracted from individual images, the task of finding the correspondence between cells at different time points, so that temporal developmental signal can be extracted for each cell, remains. This is the problem that we will address in this article.

\subsection{Background}

In general, the problem of finding correspondences or matches between image objects is a fundamental problem in 
computer image analysis. In its worst case (when each object in one image can potentially correspond to every object in another image) this problem may be NP-complete. In practice this means that in order to find optimal correspondence, combinatorially many matches have to be considered. For large data sets, such a search leads to prohibitive computational times. In addition, the problem can be further complicated if unknown transformation (mapping) is applied to the objects in different image frames. In this case in addition to finding optimal correspondences between points, the transformations influencing object attributes (such as coordinates) have to be estimated as well. This is often the case in fluorescent imaging.

Many solutions to point matching and graph matching are available in the literature. The state of the art work is based on joint estimation of correspondence and spatial mappings via optimization of energy function $[5,6,7,8]$. The general framework for such optimization is proposed by Gold et al. [4]. This framework uses the methods of deterministic annealing $[9,13]$ in conjunction with soft assign $[10,14,15]$ and clocked objectives [11] to produce an optimizing network and a corresponding Energy function.

The success of such approach is dependent on the design of energy function in conjunction with the choice of optimization technique. The typical energy function consists of two parts $\mathrm{E}=\mathrm{E}_{\mathrm{p}}+\mathrm{E}_{\text {cons }}$. Energy of constrains $\left(\mathrm{E}_{\text {cons }}\right)$ is tightly related to optimization method being used, and Energy of the problem $\left(\mathrm{E}_{\mathrm{p}}\right)$ is tightly related to the problem. Ideally $\mathrm{E}_{\mathrm{p}}$ should use all the data and information available, to estimate the error of given correspondence.

Of crucial importance in designing $\mathrm{E}_{\mathrm{p}}$ is accurate selection of mapping function. If the image data has undergone affine transformation, the natural choice for mapping function is obviously an affine transformation. If the parameters of such transformation are not known, they must be estimated simultaneously with correspondence. Another example of mapping is Thin Plate Spline, proposed by Chui and Rangarajan [12] for matching objects in brain MRI. It is obvious that the $E_{p}$ part of energy function varies significantly, depending on the data and it's behavior.

\section{Problem description and objectives}

In this article, we will address tracking, matching and modeling signals in fluorescent imagery, using the latter optimizing network. More precisely, the problem is: Given a sequence of images (movie) depicting cell arrays, determine the positions of each cell at each time point (track cells through time), while recognizing cell division events and recording cell lineage. Cell death is not observed in the image data under study, however during growth cells often disappear into the "out of focus" regions.

Cell centroids and other attributes have been previously extracted from such images using various image processing algorithms as described briefly in Section 6. Extraction of cell attributes from individual images is not a focus of this paper. Instead, we concentrate on determining correspondences between already extracted cells in consecutive images. Determining such correspondences between cells can be very challenging, especially for low sampling rate, when cells move a lot from frame to frame. It often requires modeling of cell motion. In addition, the limitations of imaging process and image processing algorithms produce mistakes in extracted cell attributes, therefore making matching job even harder.

The main challenge here is to design the $\mathrm{E}_{\mathrm{p}}$ and mappings adequate for extraction and modeling of temporal cell signals. The form of some of these mappings is known a priori. For example, it is usually known that the data might undergo rotation and translation or other Euclidean or affine transformations during development. But there are other mappings present in cell colonies that are less clear and extremely important. Such mappings are caused by cell growth and cell division (growth transformation), and they are by far less known and they are actually the object of the scientific study. In fact, the growth transformation involves (is a function of) cell products and is tightly interconnected with the dynamic network of the cell colony under study. In principle, as far as cell growth and cell division transformations (mappings) are concerned, we are confronted with a "chicken and egg problem". To extract the signal from the data, one must hypothesize the mapping, but two hypothesize the mapping one must extract the signal from the data. In this way our objectives form a closed loop (Figure 1). Each node in a loop enables next node. Once again a simultaneous solution is desired, but it is only possible if the form of growth and cell division mappings can be hypothesized. Practically, this problem is solved by incremental improvement of $\mathrm{E}_{\mathrm{p}}$, and analysis of obtained solutions.

In such approach we would start with a very simple $E_{p}$, and we would apply it to the best obtainable data. Such data must be collected at the fastest sampling rate possible, so that cell displacement due to growth and cell division transformations is negligible. That allows us to ignore growth transformation, while extracting a temporal cell signal, and then to hypothesize such transformation given extracted signal. Once the form of growth transformation is hypothesized, the simultaneous solution for growth mapping and correspondence can be obtained, and then the interactions of these transformations with other cell data/parameters can be hypothesized and formulated as a dynamic network.

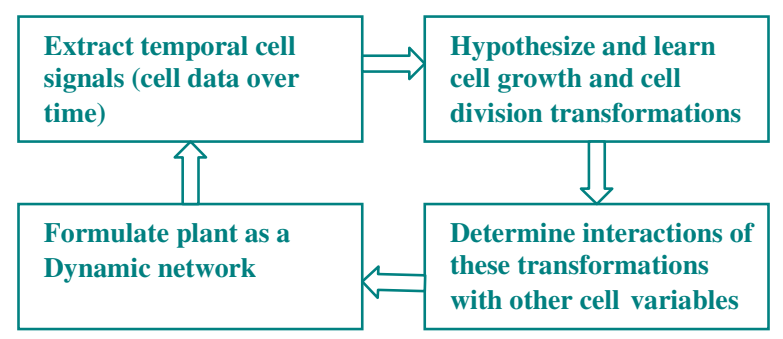

Figure 1. The objectives loop. Each objective enables the next one. 


\section{Approach to solution: Sequential vs. Simultaneous solutions}

Our general approach to the solution is to encode problem goals as terms in an objective function and to determine the point (cell) correspondence and transformation parameters, which optimize the objective function. Such optimization can be done in steps with simpler energy functions, where each optimization step assumes the results from previous optimization steps (Figure 2), or it can be done jointly with one more complicated (Eq. 3 and Eq. 5) energy function modeling all the unknown mappings.

Since after each step in sequential processing, the overall search space is significantly reduced, and since the energy functions for such processing are generally much simpler, the sequential solution is faster and easier to implement. The drawback, of course, is that not the entire search space has been evaluated and the optimal solution with respect to all variables might be less accurate. However, if the sequential process is stated in such way that most reliable optimization components are done first, the accuracy of the results in practice might even exceed the one obtained with joint optimization. Often, for easier data sets, when the displacement of the cells in consecutive images is small the sequential approach outperforms joint optimization (See Section 6). Nevertheless, this is not the case with more challenging data sets. In these cases, joint optimization tends to outperform sequential processing.

Our approach to cell tracking in fluorescent imagery will start with the simple objective functions suitable for sequential optimization (Eq.1) and (Eq.2), and it will progress to the more complicated energy functions, suitable for simultaneous optimization.

\section{Sequential Solution}

In sequential optimization the energy function is split into components and each component is optimized separately without feedback. Sequential optimization for the problem of extracting temporal cell signals from GFP data is depicted in Figure 2. This approach first determines point matching in each pair of two consecutive images. It ignores cell growth and cell division mappings and calculates correspondences using $\mathrm{E}_{\mathrm{p}}$ as in (Eq. 1).

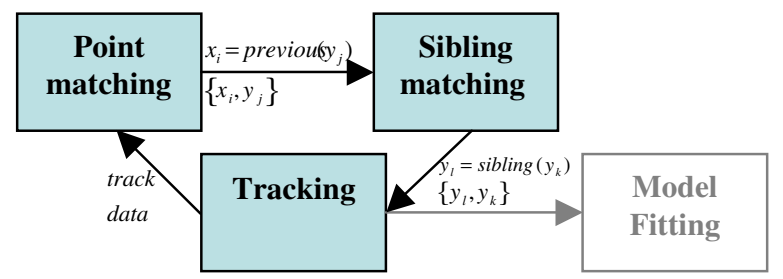

Figure 2. Sequential solution for tracking cell signals.
The correspondence matrix $M_{i j}$ includes slack variables, thereby allowing non-matches for outlier points. The output of point matching are matched pairs $\left\{x_{i}, y_{j}\right\}$ where $x_{i}$ is the point (cell) $i$ from previous image (or nothing) and $y_{j}$ is cell $\mathrm{j}$ from following image (or nothing). The overall energy function to be minimized is a total error (Euclidian distance between cell positions in consecutive images) of all matches scaled by the expected variance of this error $\left(\sigma^{2}\right)$, and is given by (Eq. 1). The mapping is assumed to be affine (with parameters $A$ ) and is known for some of the data. When it is not known, $A$ is optimized jointly with correspondence [4].

$$
E p=M_{i j, j=1}\left(\left\|A x_{i}-y_{j}\right\|^{2} / \sigma^{2}\right) \quad \text { (Eq.1) }
$$

Because of cell divisions, which are frequent in our microscope data, the previous image in the sequence contains smaller number of cells then following image. Therefore, there usually will be a number of $y_{k}$ that matched to nothing in previous image. Such $y_{k}$ are deduced with high probability to be a product of cell division. The next step in sequential approach is to determine parents and siblings for such $y_{k}$ (given found matched pairs). The objective function for this process is given by $E_{s}$ in (Eq. 2). The first term is the Euclidian distance between two siblings from the same image. It is scaled by expected variance of this distance $\left(\sigma_{(1)}^{2}\right)$. The second term is the Euclidian distance between $y_{k}$ and the parent of it's sibling $y_{l}$ scaled by expected variance of this distance $\left(\sigma_{(2)}^{2}\right)$. The objective function is defined to minimize total error between current cells and their siblings and between current cells and sibling's parents. The correspondence matrix entry $L_{k l}$ will optimize to 1 , if cells $y_{k}$ and $y_{l}$ are siblings with common parent in the previous image, and it will optimize to 0 otherwise. Just like $M_{i j}, L_{k l}$ includes slack row and column for possible non-matches. Even though in our data sets cells are not expected to dye or appear out of nowhere (orphans), this often happens in real imagery due to imaging limitations. For future reference, we always include slack row and column in all correspondence matrices mentioned in the rest of this article.

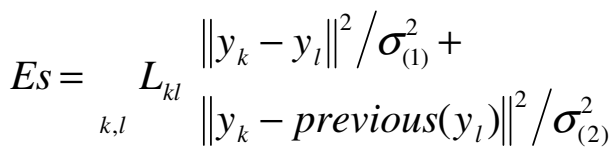

The remaining component of the sequential solution is tracking. In general, tracking combines pairwise matches to produce the tracks (paths) of the cells through the entire image sequence (movie) and builds the lineage tree. If the cells move randomly in the image and do not follow any trajectory that can be modeled, or if such trajectory is not immediately observable (as is often the case with sequential approach), not much can be gained from tracking in terms of accuracy of the results. In this case, pairwise matches are given a priori, and tracking is just a greedy search 
implemented via simple "bookkeeping" algorithm. The only improvement this algorithm makes in terms of accuracy is some inference of missing or merged cells, resulted from the mistakes of cell extraction algorithms. The result of the tracking algorithm is a list of tracks (track data), each track specifying the coordinates and attributes of one cell in all images it exists in. Once such tracks are extracted and the attributes of cells over time can be quantitatively examined, the model for cell motion and growth can be assumed and fitted into existing data (Model Fitting step).

\section{Simultaneous solutions}

\subsection{Pairwise matching with cell division}

In simultaneous solution the components of sequential solution are combined in joint optimization. We first attempt to perform joint optimization of cell matching and sibling matching. We use Robust Point Matching algorithm utilizing Thin Plate Spline (TPS) transformations with softassign embedded in deterministic annealing loop [12]. The full objective function ( $\mathrm{E}=\mathrm{E}_{\mathrm{p}}+\mathrm{E}_{\text {const }}$ ) in such matching problem is given in (Eq.3)

$$
\begin{aligned}
& E=M_{i, \alpha, j} \theta-\frac{\left[\left|x_{i, \alpha}-\varpi\left(y_{j}\right)\right|^{2}\right]}{\sigma^{2}} \\
& -\frac{1}{\beta} \quad M_{i, \alpha, j}\left(\log M_{i, \alpha, j}\right) \\
& -\lambda_{1} \operatorname{Tr}\left(\omega^{T} \hat{\Phi} \omega\right)-\lambda_{2} \operatorname{Tr}\left[(d-\hat{I})^{T}(d-\hat{I})\right] \\
& -\mu_{k} M_{r(k)} M_{i, \alpha, j}
\end{aligned}
$$

The $M_{i, \alpha, j}$ are several matrices with $\alpha=\{1\}, \alpha=\{1,2\}$, or $\alpha=\{1,2,3\}$, obeying doubly stochastic rules $\mathrm{r}(\mathrm{k})$ as described in Section 5.3. The $\Phi\left(y_{j}\right)$ is thin plate spline transformation of the vector $y_{j}$ as shown in (Eq. 4)

$$
\varpi\left(y_{j}\right)=y_{j} d+\phi\left(y_{j}, y_{k}\right) \omega_{k} \quad \text { (Eq.4) }
$$

Here affine matrices $d$ and warping vectors $\omega_{k}$ constitute the TPS coefficients, and $\phi$ is the corresponding kernel function. $\hat{\Phi}$ is composed of $\phi\left(y_{j}, y_{k}\right)$ values of the kernel function. The first term of this function is the Euclidian distance error of hypothesized correspondence. Parameter $\theta$ regulates the probability of cell matching to nothing. Increasing $\theta$ decreases the probability of non-matches during stochastic optimization and vise versa. The third term in $E$ is the standard thin-plate spline regularization term which penalizes the local warping coefficients $\omega$. $\square$ The forth term constraints affine mapping $d$ by penalizing the residual part of $\mathrm{d}$ which is different from an identity matrix I. $\lambda_{2}$ and $\lambda_{1}$ coefficients penalize the affine and warping parts of the TPS accordingly. Normally $\lambda_{2}$ is set small to adjust the affine coefficients $d$ before $w$. The second and fifth terms of the energy function represent the constraints imposed on the problem $\left(\mathrm{E}_{\text {const }}\right)$. The second term is an entropy barrier function with the temperature parameter $\mathrm{T}=1 / \beta$. It is used in deterministic annealing step [9]. The fifth term is a stochastic optimization term with the Lagrange parameters $\mu_{k}$ corresponding to the rules $\mathrm{r}(\mathrm{k})$ realized by softassign [10]. The details of novel normalization rules for this pairwise matching are presented in Section 5.3.

\subsection{Tracking with cell division and affine transformation}

Finally, we attempt to combine all steps of sequential process in one joint optimization. This is achieved with the Energy function in (Eq. 5).

$$
\begin{aligned}
& L_{\alpha, i}^{t}\left[\mid x_{i}^{t}-y_{\alpha}^{t} \|^{2} / 2 \sigma_{(1)}^{2}-\theta\right] \\
& E=\underset{t}{e^{-\lambda t}+}{ }_{\alpha=1}\left\|y_{\alpha}^{t+1}-A^{t} \bullet y_{\alpha}^{t}\right\|^{2} / 2 \sigma_{(2)}^{2} \\
& +\underset{\alpha, \beta=1}{N_{\beta \alpha}^{t}}\left[\left\|y_{\beta}^{t+1}-A^{t} \bullet y_{\alpha}^{t}\right\|^{2} / 2 \sigma^{2}-\bar{\theta}\right]
\end{aligned}
$$

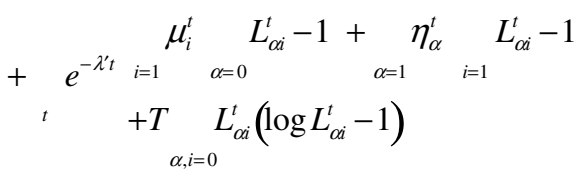

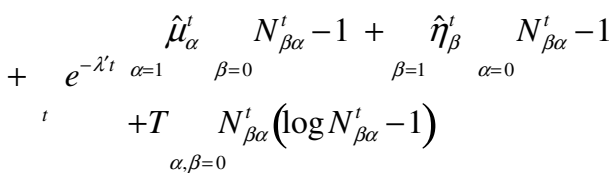

This energy function is minimized by estimating track coordinates at time $\mathrm{t}\left(y_{\alpha}^{t}\right)$ and affine transformation $A^{t}$, while minimizing: total error between estimated tracks and true point coordinates at time $\mathrm{t}\left(x_{i}^{t}\right)$ (first term of first sum); total error between consecutive points of each track, given estimated affine (second term of first sum); total error between sibling track $\left(y_{\beta}^{t}\right)$ and it's transformed parent (third term of first sum). The remaining two sums of this energy function constitute $\mathrm{E}_{\text {cons }}$ (the energy of constrains). As before, there is an entropy barrier function terms (last terms of second and third sums) with annealing temperature $\mathrm{T}$; and unique correspondence optimization terms with Lagrange parameters $\mu_{i}^{t}$ and $\eta_{\alpha}^{t}$ for the correspondence between tracks and points at time $\mathrm{t}\left(L_{\alpha i}^{t}\right)$, and with Lagrange parameters $\hat{\mu}_{\alpha}^{t}$ and $\hat{\eta}_{\beta}^{t}$ for the correspondence 
between siblings at time $\mathrm{t}\left(N_{\beta \alpha}^{t}\right)$. Note that this energy function has several correspondence problems to solve simultaneously: $L_{\alpha i}^{t}$ and $N_{\beta \alpha}^{t}$ for every time point $t$. Solving for $L$ and $N$ performs joint optimization for track and sibling matching, and optimizing these matrices jointly for every time point $t$ performs joint optimization of tracks. We included entropy and unique correspondence constraints for each correspondence problem and scaled the energy function with $e^{-\lambda t}$, thus placing more weight on earlier matches to insure forward directionality of the solution dynamics.

As was stated in the background section the joint optimization is made possible by softassign, deterministic annealing and clocked objectives methods. The softassign is based on Sinkhorn's theorem [16], which states that a doubly stochastic matrix is obtained from any positive square matrix by alternating row and column normalizations. Such a normalization process is directly related to solving for Lagrange multiplier parameters. The original entries of the stochastic matrix (in this approach $L$, and $N$ ) are typically an error term $Q_{i j}$ obtained by setting the partial derivatives of Energy function $\mathrm{E}$ with respect to correspondence matrices to zero $\left(\partial E / \partial L_{\alpha i}^{t}=0\right.$ and $\partial E / N_{\beta \alpha}^{t}=0$ ). If original $Q_{i j}>0$, the softassign insures that

$$
Q_{i j} \approx 1 \text { for all } \mathrm{i} \text { and } Q_{i j} \approx 1 \text { for all } \mathrm{j} \text {. Softassign in }
$$
$j$

conjunction with deterministic annealing can find global minimum in the assignment problem [9]. As the temperature is reduced the doubly stochastic matrix approaches a permutation matrix imposing an additional constraint $Q_{i j} \approx \begin{aligned} & 0 \\ & 1\end{aligned}$.

To obtain closed form solutions for unknown parameters the method of Clocked Objectives is used. In this method the unknown parameters are obtained by differentiating energy function with respect to these parameters and setting result to 0 (to find the minimum). The closed-form solutions are obtained in the iterative scheme [11]. The iterative scheme for the given problem is represented by formula in (Eq.6). This formula states that in iterative scheme first the track values $y_{\alpha}^{t}$ have to be estimated by calculating an analytical solution to $\partial E / \partial y_{\alpha}^{t}=0$, then assuming found track values; the transformation matrix $A^{t}$ has to be estimated by calculating an analytical solution to $d E / \bar{d} A^{t}=0$. Then assuming the current estimates for track values and affine transformation, the correspondence matrices $L_{\alpha i}^{t}$ and $N_{\beta \alpha}^{t}$ are determined by solving for Lagrange multipliers $\mu, \eta, \hat{\mu}, \hat{\eta}$ via previously described softassign technique.

$F_{\oplus}=F\langle y, A,\langle(L, \mu),(L, \eta\rangle,\langle(N, \hat{\mu}),(N, \hat{\eta}\rangle\rangle \quad(E q .6)$

\subsection{Normalization rules for pairwise matching}

In order to facilitate Robust Point Matching Thin-Plate Spline (RPM-TPS) algorithm [12] for more than one possible mapping several correspondence matrices have been used (indexed by $\alpha$ ). One matrix representing a usual one-to-one match, and the other two, similarly, representing possible split into a one-to-two match (See Figure 3). The exclusion requirement has been implemented through modified row and column unique correspondence constraints.

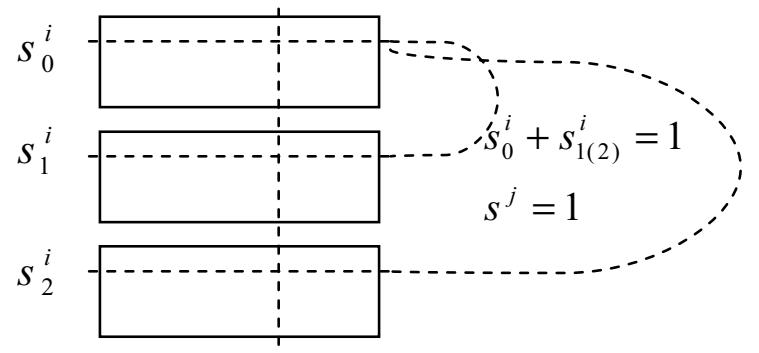

Figure 3. Paired stochasticity of corresponding joined rows. $s_{0}^{i}, s_{1}^{i}$, and $s_{2}^{i}$ are corresponding summations for the three matrices.

As before, a unique match constraint along columns insures that there is no more than one match for each point from the second set. Therefore, the normalization rule asserting unique $j$ point for each $i$ point is the same (Eq. 7).

$$
M_{i, \alpha, j}=1
$$

However, for $j$-summations a different rule is in effect. There can be either parent-to-one or parent-to-two daughter correspondences, but not both. This leads to

(Eq.8)

$$
\left.M_{i, \alpha, j}\right|_{\alpha=1}=\left.\left.M_{j}\right|_{i, \alpha, j}\right|_{\alpha=2}=1-\left.M_{i, \alpha, j}\right|_{\alpha=0} \text { (Eq.8) }
$$

If corresponding summations for the three matrices (including the slack elements) are: $s_{0}^{i}, s_{1}^{i}$, and $s_{2}^{i}$, then the above rules are satisfied by multiplying each I-raw by $\kappa_{0}^{i}, \kappa_{1}^{i}$, and $\kappa_{2}^{i}$ accordingly as in (Eq.9).

Paired constraint of corresponding joined rows from the first and the second matrices as well as the first and the third (Figure 3), insure that annealing procedure will leave either one match for a point from the first set in the upper matrices, or two possible matches on the same-indexed rows in the matrices below, but never both of them at the same time. Because each member of each matrix multiplies a separate term in the objective function (see Eq. 3), this approach provides both simultaneous solution of the split-matching problem and flexibility in coupling different points from different matching possibilities. 


$$
\begin{aligned}
& k_{0}^{i}=\frac{2}{\left(s^{i}+1 / 2\right)^{2}} \\
& k_{1}^{i}=\frac{s_{2}^{i}}{s^{i}+1 / 2} \\
& k_{2}^{i}=\frac{s_{1}^{i}}{s^{i}+1 / 2},
\end{aligned}
$$

where

$s^{i}=\sqrt{\frac{1}{4}+s_{0}^{i} /\left(s_{1}^{i}+s_{2}^{i}\right)}$

\section{Experiments}

We have used three data sets in our analysis: 3dimensional images of a growing plant shoot-apical meristem, 2-dimensional images of developing colonies of bacillus Subtillus, and 2-dimensional images of developing colonies of e-coli. The extraction of cell coordinates from 3$\mathrm{d}$ images has been performed via standard gradient descent method by the team of scientists from Lund University, Sweden, under the leadership of Henrik Jönsson. The extraction of cell coordinates and other cell attributes from 2-d images have been previously accomplished by the Caltech team under the leadership of Michael Elowitz. They used iterative erosion/dilation algorithms for this purpose.

The e-coli and bacillus Subtillus data sets are 2dimensional. It is possible to visualize and ground truth these data (Figure 4, Figure 5). The bacillus Subtillus and ecoli are not rigidly attached to each other and can move noticeable distances from frame to frame. Such motion is the main challenge of these data sets.

The shoot apical meristem data is 3-dimensional. In this data, cell walls are attached to each other, and interact mechanically preventing large displacements. In this data cells appear to move outwards (Figure 6b)), but such motion can be observed only over large time lapses (about 10 time points); locally cell displacements appear random. This can be modeled as an additional track constraint in the joint track optimization method. It is more difficult to visualize and ground-truth this data. To evaluate roughly the performance of this data track statistics and visualization tools have been used.

Currently, tracking (matching all pairs of images simultaneously as in Eq. 5) suffers from track fragmentation (breaking up of one cell track into few segments). The main reason for this is that number of tracks has to be significantly larger then number of cells in individual image (especially in the beginning of the sequences). Therefore, for the goodness of match based on Euclidian distance, it is less costly to fit few tracks into set of points, rather then one. We attempted to control this problem with slack parameter $\theta$ and by adding of an additional term (the squared sum of correspondence matrix entries, excluding slacks), but did not get much more control over the problem.
This study still continues. Therefore for comparison of sequential and simultaneous methods, we focus on sequential approach in Eq. 1) and Eq. 2) and joint optimization approach in Eq. 3).

In bacillus Subtillus data, estimating track values via sequential approach slightly outperformed the joint optimization approach. In joint optimization approach 68 points were matched wrong out of total 2217 points collected from 22 images, therefore amounting to 3\% error. In sequential optimization (pairwise matching with TPS) approach 40 points were mismatched, therefore amounting to $1.8 \%$ error.

Figure 4 depicts the tracking of one colony (out of 6 ) on 22 consecutive images (only 4 early consecutive images are displayed here due to the lack of space). The corresponding cells are numbered with the same track number. The track identification numbers of found siblings are displayed in parenthesis under current track number.

In e-coli data (Figures 5b), 5c)), the joint optimization approach overall outperforms sequential approach, producing $5.35 \%$ error vrs. $6.44 \%$ error (in sequential approach). The e-coli data is different from bacillus Subtillus data, it is complicated by the presence of long irregular cells as in Figures 6b) and 6c). The energy functions were mainly designed for Subtillus data, without consideration for long irregular cells, therefore the worst performance has been expected. However, the joint optimization method demonstrated to be more robust by outperforming sequential method on this difficult data. Figure 6a) depicts the percentage error for 35 pairwise matches (36 individual images). Since the total number of cells in each image of the sequence is different there is no direct relationship between overall error and error/per image. For example, if some early image contains two cells, one of which is matched wrong, there will be $50 \%$ error for this image, but overall percent error increase will be negligible. The results of sequential optimization are displayed with dashed line, and the results of joint optimization are depicted with solid line. Note that both algorithms resulted with 0 error for first 25 images, however the error rates grow with complexity (number of cells) and an increase in missed (by segmentation process) cells.

Another interesting factor to observe is that the errors produced by sequential and joint optimization approaches are somewhat orthogonal, meaning that the erroneous matches seem to be different for both approaches. In sequential approach most of the errors were produced in matching of the siblings: $11.9 \%$ error for sibling matching, $4.2 \%$ error for same cell matching. In joint optimization approach $4.2 \%$ error was accomplished in sibling matching, and $5.8 \%$ error was produced in same cell matching. Moreover, joint optimization errors appear to be of global character. For example, note erroneous track 78, such mismatch is easily detected with a sanity test based on distance only. Sequential optimization errors are mainly local. Note erroneous tracks 3 and 54. This leads to the possibility that overall results can be improved by combining the results of both algorithms. 
Finally, we present some preliminary results of tracking signal in shoot apical meristem data. In the absence of ground-truth, we have used statistics to select reasonable solutions. Such statistics include the average density of tracks and the average standard deviation of tracks, which are inversely related; and also include the number of orphans (newly appearing cells without matched parent) and number of deaths (disappeared cells). The latter two measures ideally should be very small, but such criterion cannot be enforced rigidly. Extracting these statistics under present conditions assists in selecting reasonable solutions, but is not sufficient for comparative analysis.

One such solution is depicted in Figure 5. For reasons of legibility only few tracks are included in the plot of Figure 5-a). The star denotes the beginning of the track, and branches represent cell divisions. The newly born cells are connected to their parents with black dashed lines. One can observe that cell motion appears locally random, but a global outward growth tendency is observed as well. A clearer view of an outward growth tendency form an interpolated total displacement field for 10 time points, is depicted in Figure 5-b).
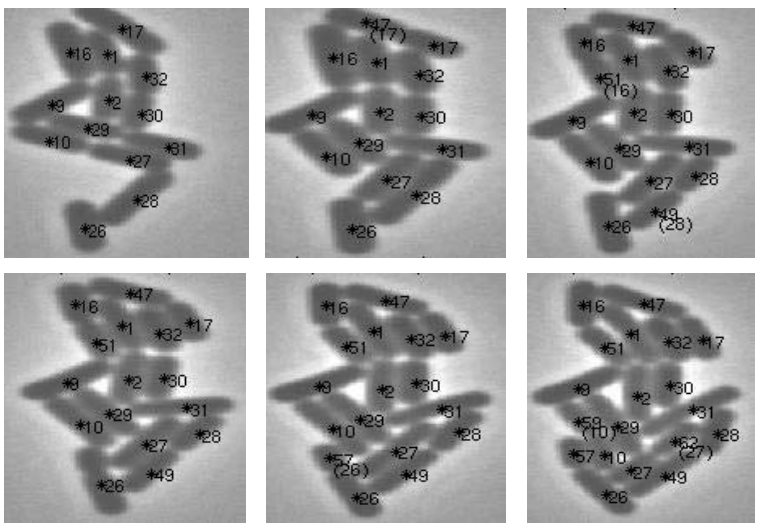

Figure 4. Pairwise matching for tracking bacillus Subtillus data.

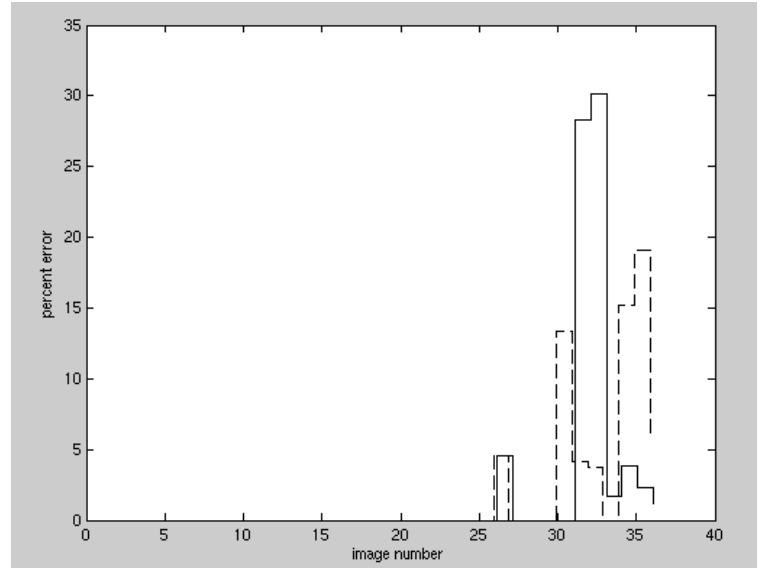

a) Percentage error for 35 pairwise matches produced by sequential optimization approach (dashed line) and joint optimization approach (solid line).
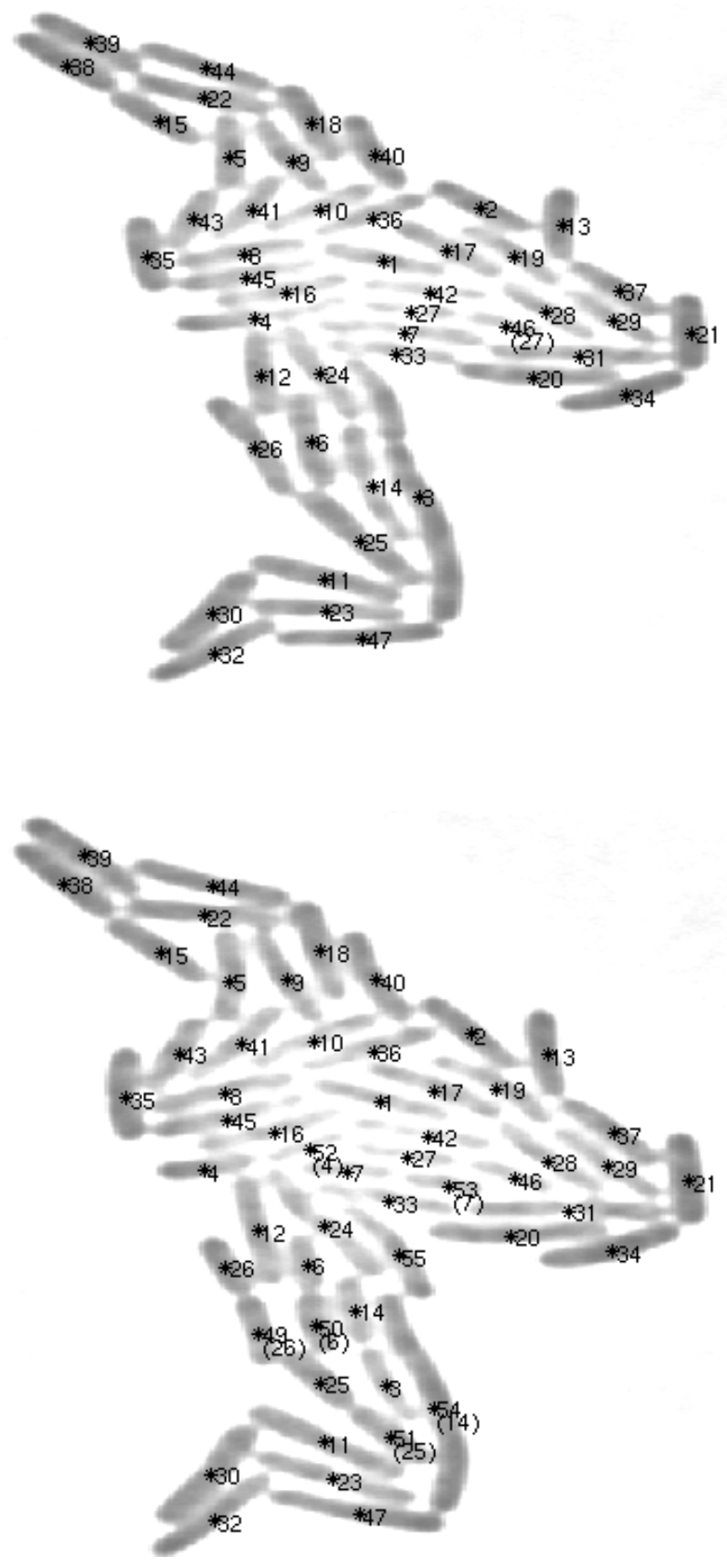

b) Matching cells in e-coli images $31 / 32$ with sequential optimization approach. Matches displayed on raw image data. 

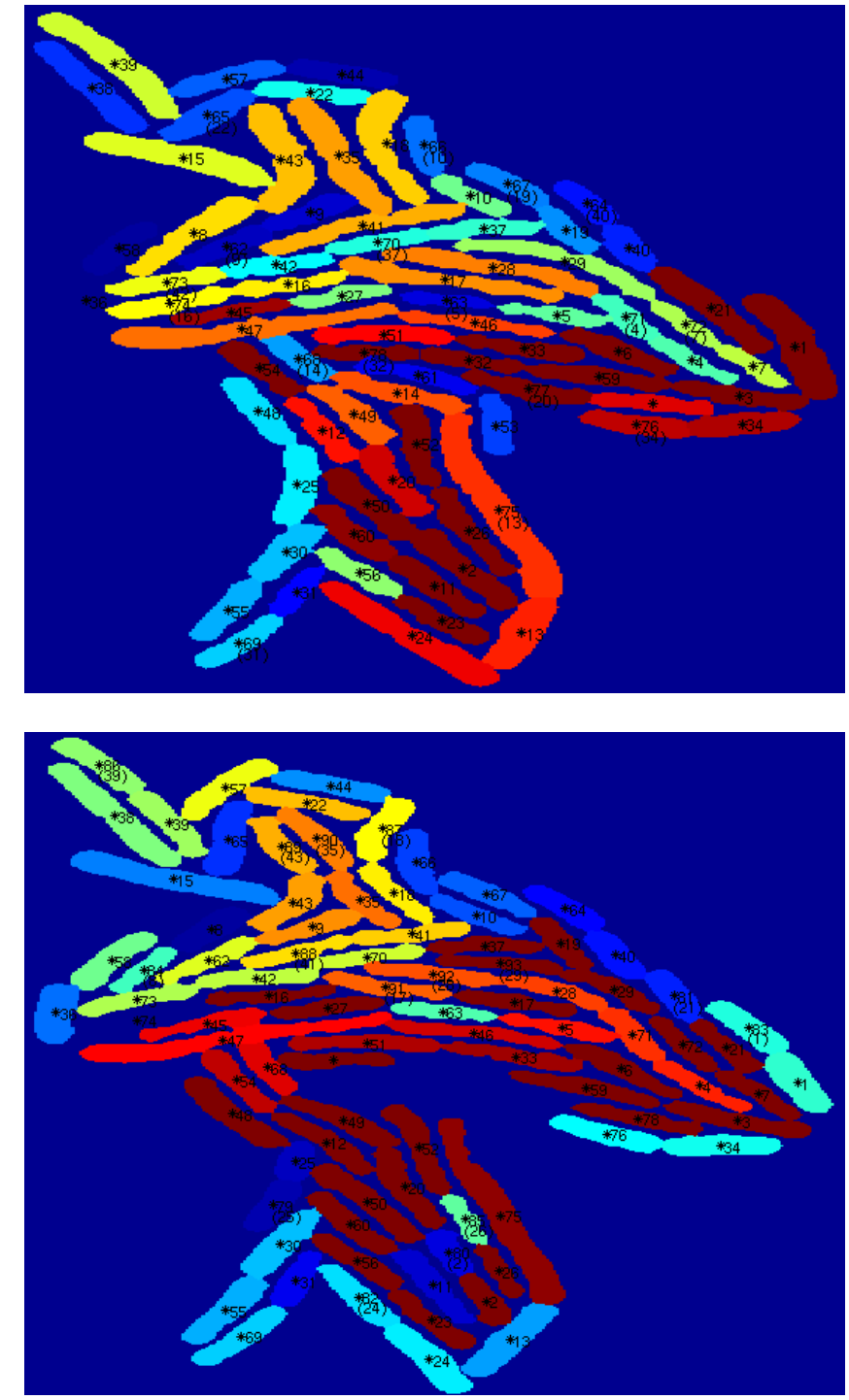

c) Matching cells in e-coli images $34 / 35$ with joint optimization approach. Matches displayed on segmented image data.

Figure 5. Sequential vrs. joint optimization in e-coli data.

\section{Conclusions and plans}

In this paper we have demonstrated sequential and joint optimization techniques applied to tracking cell signals in GFP images. There are two main challenges that such tracking has to confront. The first challenge is matching allowing one-to-two correspondences. We have addressed this challenge with an extended softassign algorithm, which performs well and presents a valuable general tool for the solution of one-to-many correspondences. The second challenge is missing cells mistakes produced by cellsegmentation. Some of these mistakes were addressed successfully via track inference in the sequential approach. The more general approach, which performs joint estimation of track values, can handle missing cells better, but it suffers from track fragmentations. Such an approach is more important when cell motion has structure or can be expressed as a function of cell variables. Therefore, it is more likely to be used in the final dynamic model. In the future, we plan to deal with cell segmentation (extraction) mistakes by performing cell segmentation and cell matching jointly. This is especially useful since there is no automatic way to assess the goodness of the segmentation, but there is a way to assess goodness of the match (number of unmatched points).

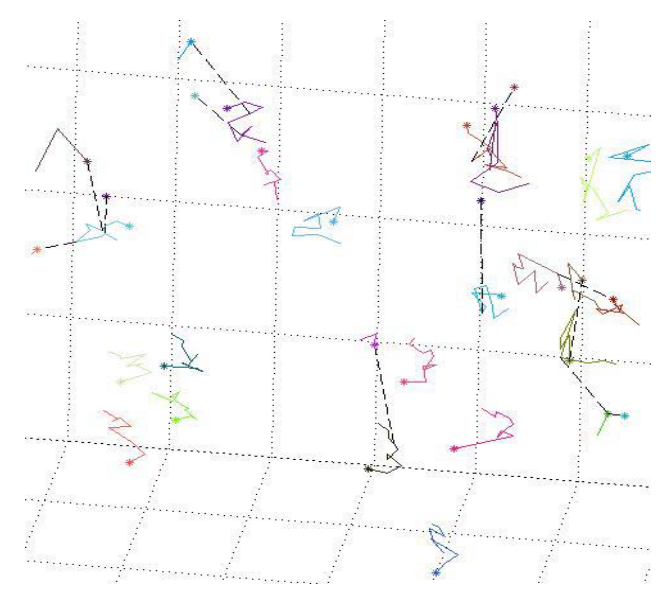

a) Selected tracks generated for shoot apical meristem data.

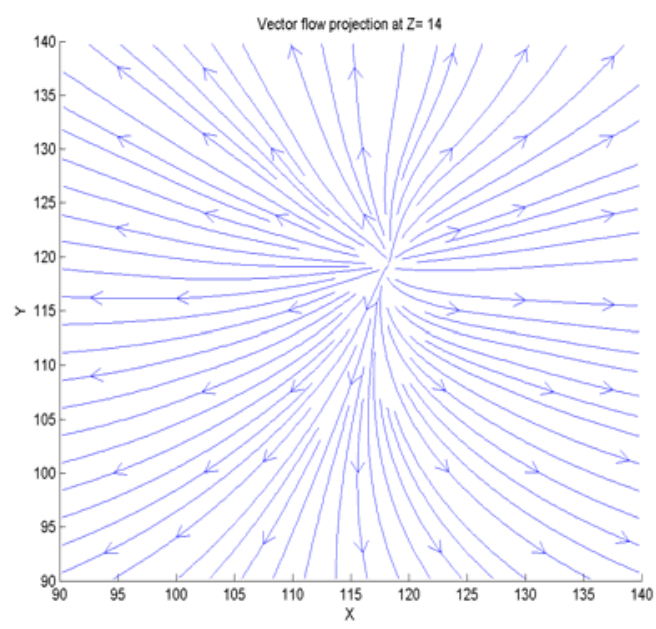

b) The interpolated displacement field for large time step.

Figure 6. Tracking Plant Shoot Apical Meristem Data.

Our current software is used by biologists to extract the cell signals from GFP imagery. Given such signals, scientists are able to hypothesize the details of underlying cell processes, to model these processes in dynamic networks and to make predictions about organism behavior. 
On the other hand, such models and predictions allow for better signal extraction (tracking algorithms), producing more accurate data for use in biological research.

\section{Acknowledgements}

This work was supported by NSF:FIBR award number EF-0330786. We are grateful to Marcus Heisler, Elliot M. Meyerowitz, Henrik Jönsson, and Joe Roden for their roles in providing test data.

\section{References}

[1] Henrik Jönsson, Marcus Heisler, G. Venugopala Reddy, Vikas Agrawal, Victoria Gor, Bruce E. Shapiro, Eric Mjolsness, Elliot M. Meyerowitz, "Modeling the organization of the WUSCHEL expression domain in the shoot apical meristem", Bioinformatics, vol.00, no.00, 2005.

[2] Luc Vincent and Pierre Soille, "Watersheds in digital spaces: an efficient algorithm based on immersion simulations", IEEE Trans. Patt. Anal. Mach. Intell., vol.13, no.6, June 1991.

[3] Pierre Soille, Edmond J. Breen, Ronald Jones, "Recursive implementation of erosions and dilations along discrete lines at arbitrary angles, IEEE Trans. Patt. Anal. Mach. Intell., vol.18, no.5, may 1996.

[4] Steven Gold, Chien- Ping Lu, Anand Rangarajan, Suguna Pappu, and Eric Mjolsness,'New algorithms for 2-D and 3-D point matching: pose estimation and correspondence", Pattern Recognition, 1998.

[5] S. Gold and A. Rangarajan, "A graduated assignment algorithm for graph matching”, IEEE Trans. Patt. Anal. Mach. Intell., 1996.
[6] A. D. J. Cross and E. R. Hancock, "Graph matching with a dual-step EM algorithm.”, IEEE Trans. Patt. Anal. Mach. Intell., 1998.

[7] S. Sclaroff and A.P. Pentland, "Model matching for correspondence and recognition", IEEE Trans. Patt. Anal. Mach. Intell., 1995.

[8] Anand Rangarajan, Haili Chui, Eric Mjolsness, Suguna Pappu, Lila Davachi, Patricia S. Goldman-Rakic, and James S. Duncan,

"A robust point matching algorithm for autoradiograph alignment", Medical Image Analysis, 1997.

[9] J.J. Koslowsky and A.L. Yuille, "The invisible hand algorithm: Solving the assignment problem with statistical physics", Neural Networks, 1994.

[10] S. Gold and A. Rangarajan, "Softmax to Softassign: Neural network algorithms for combinatorial optimization", Journal of Artificial Neural Networks, 1996.

[11] E.Mjolsnessand W. Miranker, "A Lagrangian Formulation of Neural Networks II: Clocked Objective Functions and Applications", Neural, Parallel and Scientific Computations, vol 6 no. 3, pp. 337-372, 1998.

[12] H. Chui and A. Rangarajan, "A new algorithm for Non-Rigid Point Matching”, CVPR, 2000.

[13] A.L. Yuille, J.J Koslowsky, "Statistical physics algorithms that converge", Neural Computation, 1994.

[14] D.E. Van den Bout, T.K. Miller, "Improving the performance of the Hopfield-Tank neural network through normalization and annealing", Biological Cybernetics, 1989.

[15] P.D. Simic, "Statistical mechanics as the underlying theory of 'elastic' and 'neural' optimizations", Network, 1990.

[16] R.Sinkhorn, “A relationship between arbitrary positive matrices and doubly stochastic matrices", Annals of Mathematical Statistics, 1964. 